\title{
Validation of Soil Test and Yield Target Based Fertiliser Prescription Model for Hybrid Maize on Vertisol
}

\author{
R. Suresh ${ }^{*}$ and R. Santhi \\ Department of Soil Science and Agricultural Chemistry, Tamil Nadu Agricultural University, \\ Coimbatore-641 003, Tamil Nadu, India \\ *Corresponding author
}

\begin{tabular}{|c|}
\hline Keywords \\
\hline $\begin{array}{l}\text { Vertisol, Maize, } \\
\text { STCR-IPNS, } \\
\text { Validation and } \\
\text { Yield }\end{array}$ \\
\hline Article Info \\
\hline $\begin{array}{l}\text { Accepted: } \\
\text { 16 August } 2018 \\
\text { Available Online: } \\
\text { 10 September } 2018\end{array}$ \\
\hline
\end{tabular}

\section{A B S T R A C T}

Field experiments were conducted at three different locations in southern zone of Tamil Nadu to validate the fertiliser prescription developed from Soil test Crop Response correlation under Integrated Plant Nutrition System (STCR-IPNS) for maize on vertisol based on Inductive cum Targeted yield model. The treatments include control, blanket recommendation (100\% RDF), blanket recommendation (NPK+FYM @ 12.5t ha ${ }^{-1}$ ), soil test crop response (STCR) based fertilizer dose for an yield target of 9.0, 10.0 and $11.0 \mathrm{t}$ $\mathrm{ha}^{-1}$, STCR-IPNS based fertilizer dose for an yield target of 9.0, 10.0 and $11.0 \mathrm{t} \mathrm{ha}^{-1}$ and farmer's practice. The N, P and K fertilizer for different targets was calculated based on the initial soil test values of the respective locations. The cultivation practices were carried out periodically and the grain yield was recorded at harvest. Using the data on grain yield and fertilizer doses applied, parameters viz., per cent achievement, response ratio (RR) and benefit: cost ratio (BCR) were computed. The results revealed that the targeted yield has been achieved within $+/-10$ per cent variation proving the validity of the equations. The range and mean values of the three validation experiments indicated that the highest mean grain yield of maize was recorded with STCR-IPNS-11t ha ${ }^{-1}\left(11.08 \mathrm{tha}^{-1}\right)$ followed by STCR-IPNS-10 tha ${ }^{-1}\left(10.15 \mathrm{t} \mathrm{ha}^{-1}\right)$. Though the highest response ratio of $14.44 \mathrm{~kg} \mathrm{~kg}^{-1}$ was recorded in STCR-IPNS-9 $\mathrm{t} \mathrm{ha}^{-1}$, the highest BCR was recorded in STCR-IPNS-11 $\mathrm{tha}^{-1}$ (2.26). The mean increase in yield due to STCR-IPNS-11 t ha ${ }^{-1}$ was 39.7 per cent over blanket (100\% RDF), 17.6 per cent over blanket + FYM@ 12.5t ha ${ }^{-1}$ and 59.6 per cent over farmer's practice. Farmer's practice recorded relatively lower yield and response ratio as compared to blanket and STCR treatments while the STCR-IPNS treatments recorded the highest per cent achievement and response ratio over farmer's practice. Therefore the fertilizer prescription equations developed for hybrid maize on Pilamedu soil series (Typic Haplustert) for achieving $11 \mathrm{t} \mathrm{ha}^{-1}$ can be adapted to similar and allied series in other agroclimatic zones of Tamil Nadu.

\section{Introduction}

Fertilizer is one of the key inputs for achieving the estimated food grain production of our country. The indiscriminate use of fertilizers by the farmer without knowing the crop requirement and fertility status of the soil leads to the adverse effect on soil health and crop productivity. The escalating cost coupled with increasing demand on inorganic 
fertilizers and depletion of soil health necessitates the safe and efficient method of fertilizer application. Maize (Zea mays L.), one of the important millet crops grown in India is an exhaustive crop and the nutritional requirement cannot be met with the native nutrient reserves. Therefore, additional nutritional requirement can be met by external fertilizer application. Soil testing is one of the most efficient tool for optimum as well as balanced fertilization (Kumar et al., 2013). To determine the optimum fertilizer doses, the most appropriate method is to apply fertilizer doses based on soil test crop response under Integrated Plant Nutrition System studies (Singh et al., 2012). Soil testing becomes one of the vital tool for providing optimum dose of fertilizers to crops, thus maintaining the soil fertility. Soil test based fertilizer prescription eliminates over or under usage of fertilizers thereby increasing the fertilizer use efficiency and yield of crops. The Inductive cum Targeted yield concept developed by Ramamoorthy et al., (1967) established the theoretical basis and experimental technique to suit the Indian soil and climatic conditions. The fertilizer prescription developed from soil test crop response under Integrated Plant Nutrition System is to be validated at farmer's holdings on similar soil series. With this background, the present investigation was undertaken to validate the fertiliser prescription equations developed for maize on Pilamedu series (Typic Haplustert) at northwestern and southern zones of Tamil Nadu.

\section{Materials and Methods}

Field experiments were conducted during Kharif 2017 to validate the fertilizer prescription equation developed for hybrid maize (TNAU maize hybrid $\mathrm{CO}$ 6) at three locations, viz. Kottur Avarampatti village of Dindigul district (Southern zone), Devaiyur of Perambalur district (North western zone) and Bathrakalipuram of Theni district (Southern zone). The fertilizer prescription equations developed for desired yield target of maize for Pilamedu soil series are furnished below.

\section{STCR-NPK alone}

$\mathrm{FN}=3.78 \mathrm{~T}-0.78 \mathrm{SN}$

$\mathrm{F}_{2} \mathrm{O}_{5}=1.47 \mathrm{~T}-2.02 \mathrm{SP}$

$\mathrm{F} \mathrm{K}_{2} \mathrm{O}=1.79 \mathrm{~T}-0.14 \mathrm{SK}$

\section{STCR-IPNS (NPK+FYM)}

$\mathrm{FN}=3.78 \mathrm{~T}-0.78 \mathrm{SN}-0.89 \mathrm{ON}$

$\mathrm{FP}_{2} \mathrm{O}_{5}=1.47 \mathrm{~T}-2.02 \mathrm{SP}-0.91 \mathrm{OP}$

$\mathrm{FK}_{2} \mathrm{O}=1.79 \mathrm{~T}-0.14 \mathrm{SK}-0.62 \mathrm{OK}$

Where, $\mathrm{FN}, \mathrm{FP}_{2} \mathrm{O}_{5}$ and $\mathrm{FK}_{2} \mathrm{O}$ are fertilizer $\mathrm{N}$, $\mathrm{P}_{2} \mathrm{O}$ and $\mathrm{K}_{2} \mathrm{O}$ in $\mathrm{kg} \mathrm{ha}^{-1}$ respectively. $\mathrm{T}$ is the yield targeted in $\mathrm{q} \mathrm{ha}^{-1}$; $\mathrm{SN}, \mathrm{SP}$ and $\mathrm{SK}$ are soil available $\mathrm{N}, \mathrm{P}$ and $\mathrm{K}$ in $\mathrm{kgha}^{-1}$ respectively; $\mathrm{ON}, \mathrm{OP}$ and $\mathrm{OK}$ are $\mathrm{N}, \mathrm{P}$ and $\mathrm{K}$ supplied through FYM in $\mathrm{kg} \mathrm{ha}^{-1}$.

The validation experiments were laid out in Randomised block design with three replications. The treatments imposed were as follows (i).Blanket fertilizer dose (100\% RDF (250:75:75 kg N, $\mathrm{P}_{2} \mathrm{O}_{5}$ and $\mathrm{K}_{2} \mathrm{O}$ )), (ii) Blanket +FYM @12.5tha ${ }^{-1}$, (iii) STCR based fertilizer dose for an yield target of - $9 \mathrm{t} \mathrm{ha}^{-1}$ (iv) STCR based fertilizer dose for an yield target of-10t $\mathrm{ha}^{-1}$ (v) STCR based fertilizer dose for an yield target of-11 tha ${ }^{-1}$, (vi) STCR-IPNS based fertilizer dose for an yield target of $9 \mathrm{t} \mathrm{ha}^{-1}$, (vii) STCR-IPNS based fertilizer dose for an yield target of $10 \mathrm{t} \mathrm{ha} \mathrm{ha}^{-1}$, (viii) STCR-IPNS based fertilizer dose for an yield target of $11 \mathrm{t}$ $\mathrm{ha}^{-1}$, (ix) farmer's practice and (x) Absolute control. Initial soil samples were collected in each location and analysed for alkaline $\mathrm{KMnO}_{4}-\mathrm{N}$ (Subbiah and Asija, 1956), Olsen-P (Olsen et al., 1954) and $\mathrm{NH}_{4} \mathrm{OAc}-\mathrm{K}$ (Hanway and Heidal, 1952). The fertility status of the soil indicated that the $\mathrm{KMnO}_{4}-\mathrm{N}$ ranged from 175 to $198 \mathrm{~kg} \mathrm{ha}^{-1}$, Olsen-P from $12-13 \mathrm{~kg} \mathrm{ha}^{-1}$ and $\mathrm{NH}_{4} \mathrm{OAc}-\mathrm{K}$ from $380-584 \mathrm{~kg} \mathrm{ha}^{-1}$ (Table 
1). Based on the initial soil test values, the fertilizer doses were calculated and applied (Table 1, 2 and 3). The test crop TNAU maize hybrid CO 6 was raised during Kharif 2017 and the grain yield was recorded at harvest. Using the data on grain yield and fertilizer dose applied, the parameters, viz., percent achievement $\{$ (yield obtained/yield targeted $) \times$ $100\}$ and response ratio (RR) were worked out (Response ratio=response in $\mathrm{kg} \mathrm{ha}^{-1} /$ quantities of fertilizer $\mathrm{N}, \mathrm{P}_{2} \mathrm{O}$ and $\mathrm{K}_{2} \mathrm{O}$ applied in $\mathrm{kg}$ $\mathrm{ha}^{-1}$ ). BCR (B: C ratio) was worked out based on the standard procedure (Gittinger, 1982). The details of fertiliser doses applied, grain yield, percent achievement, response ratio and BCR are furnished in tables 1, 2 and 3.

\section{Results and Discussion}

At Devaiyur, the highest grain yield of maize was recorded with STCR-IPNS-11 $\mathrm{t} \mathrm{ha}^{-1}$ $\left(11.19 \mathrm{t} \mathrm{ha}^{-1}\right)$ followed by STCR-IPNS-10 $\mathrm{t}$ $\mathrm{ha}^{-1}\left(10.24 \mathrm{t} \mathrm{ha}^{-1}\right)$. The highest response ratio was recorded inSTCR-IPNS-9 $\mathrm{t} \mathrm{ha}^{-1}(14.49 \mathrm{~kg}$ $\mathrm{kg}^{-1}$ ) followed by STCR-IPNS-10 $\mathrm{tha}^{-1}(14.15$ $\left.\mathrm{kg} \mathrm{kg}^{-1}\right)$ and STCR-IPNS-11 $\mathrm{t} \mathrm{ha}^{-1}(14.07 \mathrm{~kg}$ $\mathrm{kg}^{-1}$ ) while the highest BCR was recorded in STCR-IPNS-11 $\mathrm{t} \mathrm{ha}^{-1}$ (2.26). The increase in yield due to STCR-IPNS-11 t ha ${ }^{-1}$ was 39.4 per cent over blanket (100\% RDF), 17.9 per cent over blanket+FYM@12.5tha ${ }^{-1}$ and 60.3 per cent over farmer's practice (Table 1).

At Kottur Avarampatti, the highest grain yield of maize was recorded with STCR-IPNS-11 t $\mathrm{ha}^{-1}\left(11.06 \mathrm{t} \mathrm{ha}^{-1}\right)$ followed by STCR-IPNS-10 $\mathrm{t} \mathrm{ha}^{-1}\left(10.13 \mathrm{tha}^{-1}\right)$. The highest response ratio was recorded in STCR-IPNS-9 $\mathrm{t} \mathrm{ha}^{-1}(14.07 \mathrm{~kg}$ $\mathrm{kg}^{-1}$ ) followed by STCR-IPNS-10 $\mathrm{t} \mathrm{ha}^{-1}$ (13.75 $\mathrm{kg} \mathrm{kg}^{-1}$ ) and STCR-IPNS-11 $\mathrm{t} \mathrm{ha}^{-1}(13.72 \mathrm{~kg}$ $\left.\mathrm{kg}^{-1}\right)$. Similar trend of superiority was reported by Coumaravel et al., (2013) in maize, Debarathi Bhaduri and Poonam Gautam (2013) in wheat. The highest BCR was recorded in STCR-IPNS-11 $\mathrm{t} \mathrm{ha}^{-1}$ (2.28). Similar results were also reported by Manish
Singh et al., (2017). The increase in yield due to STCR-IPNS-11 $\mathrm{t} \mathrm{ha}^{-1}$ was 39.8 per cent over blanket (100\% RDF), 18 per cent over blanket+ FYM@12.5tha ${ }^{-1}$ and 59.0 per cent over farmer's practice (Table 2).

At Bathrakalipuram, the highest grain yield of maize was recorded with STCR-IPNS-11 t $\mathrm{ha}^{-1}\left(11 \mathrm{t} \mathrm{ha}^{-1}\right)$ followed by STCR-IPNS-10 $\mathrm{t}$ $\mathrm{ha}^{-1}\left(10.1 \mathrm{t} \mathrm{ha}^{-1}\right)$. The highest response ratio was recorded in STCR-IPNS-9 $\mathrm{t} \mathrm{ha}^{-1}(14.79 \mathrm{~kg}$ $\left.\mathrm{kg}^{-1}\right)$ followed by STCR-IPNS-10 $\mathrm{tha}^{-1}(14.26$ $\left.\mathrm{kg} \mathrm{kg}^{-1}\right)$ and STCR-IPNS-11 $\mathrm{t} \mathrm{ha}^{-1}(14.07 \mathrm{~kg}$ $\mathrm{kg}^{-1}$ ) while the highest BCR was recorded in STCR-IPNS-11 $\mathrm{t} \mathrm{ha}^{-1}$ (2.27). The increase in yield due to STCR-IPNS-11 $\mathrm{t} \mathrm{ha}^{-1}$ was 39.9 per cent over blanket (100\% RDF), 16.9 per cent over blanket+FYM@12.5tha ${ }^{-1}$ and 59.6 per cent over farmer's practice (Table 3 ).

The results emanated from all the locations revealed that the targeted yield has been achieved within +/- 10 per cent variation proving the validity of the equations (Table 4). Similar results were reported by Santhi et al., (2011) in beetroot and Sharma et al., (2015) for pearl millet. The range and mean values of the three validation experiments indicated that the highest mean grain yield of maize was recorded with STCR-IPNS-11 $\mathrm{t} \mathrm{ha}^{-1}(11.08 \mathrm{t}$ $\mathrm{ha}^{-1}$ ) followed by with STCR-IPNS-10 $\mathrm{tha}^{-1}$ (10.15 $\left.\mathrm{t} \mathrm{ha}^{-1}\right)$. However, the yields recorded in STCR-IPNS were on par with their respective STCR-NPK alone treatments.

Though the highest response ratio of $14.44 \mathrm{~kg}$ $\mathrm{kg}^{-1}$ was recorded in STCR-IPNS-9 $\mathrm{t} \mathrm{ha}^{-1}$, the highest BCR was in recorded in STCR-IPNS11 tha $^{-1}$ (2.26). This might be due to the better use efficiency of applied NPK fertilizers under IPNS. Similar findings were recorded by Sellamuthu et al., (2015). The mean increase in yield due to STCR-IPNS-11 t ha ${ }^{-1}$ was 39.7 per cent over blanket (100\% RDF), 17.6 per cent over blanket + FYM@12.5tha ${ }^{-1}$ and 59.6 per cent over farmer's practice. 
Table.1 Results of validation experiment on maize

Location

\begin{tabular}{|l|l|l|l|l|l|}
\hline Village & $:$ & Devaiyur & Hybrid & $:$ & TNAU Maize Hybrid CO 6 \\
\hline Block & $:$ & Perambalur & Soil & $:$ & Pilamedu series (Typic Haplustert) \\
\hline Taluk & $:$ & Perambalur & D/P: & $:$ & $\mathbf{3 1 . 0 8 . 2 0 1 7}$ \\
\hline District & $:$ & Perambalur & D/H: & $:$ & $\mathbf{1 8 . 1 2 . 2 0 1 7}$ \\
\hline
\end{tabular}

\begin{tabular}{|c|c|c|c|c|c|c|c|c|c|}
\hline \multirow[t]{2}{*}{ Sl. No. } & \multirow[t]{2}{*}{ Treatments } & \multirow{2}{*}{$\begin{array}{c}\text { FYM } \\
\left(\mathrm{t} \mathrm{ha}^{-1}\right)\end{array}$} & \multicolumn{3}{|c|}{ Fertiliser doses $\left(\mathrm{kg} \mathrm{ha}^{-1}\right)$} & \multirow{2}{*}{$\begin{array}{c}\text { Grain } \\
\text { Yield } \\
\left(\mathrm{kg} \mathrm{ha}^{-1}\right)\end{array}$} & \multirow{2}{*}{$\begin{array}{c}\text { Per } \\
\text { cent } \\
\text { achieve } \\
\text {-ment }\end{array}$} & \multirow{2}{*}{$\begin{array}{c}\text { RR } \\
\left(\mathrm{kg} \mathrm{kg}^{-1}\right)\end{array}$} & \multirow[t]{2}{*}{ BCR } \\
\hline & & & FN & $\mathrm{FP}_{2} \mathrm{O}_{5}$ & $\mathrm{FK}_{2} \mathrm{O}$ & & & & \\
\hline $\mathbf{T}_{1}$ & Blanket (100 \% RDF) & - & 250 & 75 & 75 & 8025 & - & 11.20 & 1.88 \\
\hline $\mathbf{T}_{2}$ & Blanket+FYM@12.5 $\mathrm{t} \mathrm{ha}^{-1}$ & 12.5 & 296 & 101 & 108 & 9490 & - & 11.77 & 2.05 \\
\hline$\overline{T_{3}}$ & STCR-NPK alone-9.0 $\mathrm{t} \mathrm{ha}^{-1}$ & - & 186 & 108 & 108 & 9110 & 101.2 & 13.84 & 2.04 \\
\hline $\mathbf{T}_{4}$ & STCR-NPK alone-10.0 $\mathrm{t} \mathrm{ha}^{-1}$ & - & 224 & 123 & 126 & 9885 & 98.9 & 13.40 & 2.12 \\
\hline$T_{5}$ & STCR-NPK alone-11.0 $\mathrm{tha}^{-1}$ & - & 261 & 138 & 144 & 10750 & 97.7 & 13.27 & 2.22 \\
\hline$T_{6}$ & STCR-IPNS-9.0 $\mathrm{t} \mathrm{ha}^{-1}$ & 12.5 & 140 & 82 & 75 & 9370 & 104.1 & 14.49 & 2.08 \\
\hline $\mathbf{T}_{7}$ & STCR-IPNS-10.0 $\mathrm{t} \mathrm{ha}^{-1}$ & 12.5 & 178 & 97 & 93 & 10240 & 102.4 & 14.15 & 2.18 \\
\hline$T_{8}$ & STCR-IPNS-11.0 $\mathrm{t} \mathrm{ha}^{-1}$ & 12.5 & 215 & 112 & 111 & 11188 & 101.7 & 14.07 & 2.26 \\
\hline$T_{9}$ & Farmer's Practice & 0 & 240 & 75 & 0 & 6980 & - & 10.90 & 1.60 \\
\hline $\mathbf{T}_{10}$ & Absolute control & 0 & 0 & 0 & 0 & 3546 & - & - & 1.08 \\
\hline & & & & \multicolumn{2}{|c|}{ SEd } & 268 & & & \\
\hline & & & & \multicolumn{2}{|c|}{$\mathrm{CD}(\mathrm{P}=0.05)$} & 563 & & & \\
\hline
\end{tabular}

*maintenance dose

\begin{tabular}{|l|l|l|l|}
\hline Fertilizer Prescription equations & \multicolumn{3}{|c|}{ Major Nutrients $\left(\mathrm{kg} \mathrm{ha}^{-1}\right)$} \\
\hline $\mathrm{FN}=3.78 \mathrm{~T}-0.78 \mathrm{SN}-0.890 \mathrm{~N}$ & $\mathrm{KMnO}_{4}-\mathrm{N}$ & $:$ & 198 \\
\hline $\mathrm{FP}_{2} \mathrm{O}_{5}=1.47 \mathrm{~T}-2.02 \mathrm{SP}-0.910 \mathrm{P}$ & Olsen-P & $:$ & 12 \\
\hline $\mathrm{FK}_{2} \mathrm{O}=1.79 \mathrm{~T}-0.14 \mathrm{SK}-0.620 \mathrm{~K}$ & $\mathrm{NH}_{4} \mathrm{OAc}-\mathrm{K}$ & $:$ & 380 \\
\hline
\end{tabular}


Table.2 Results of validation experiment on Maize

\begin{tabular}{|l|l|l|l|l|l|}
\hline Location & \multicolumn{5}{|l}{} \\
\hline Village & $:$ & KotturAvarampatti & Hybrid & $:$ & TNAU Maize Hybrid CO 6 \\
\hline Block & $:$ & Dindigul & Soil & $:$ & Pilamedu series (TypicHaplustert) \\
\hline Taluk & $:$ & Dindigul & D/P: & $:$ & $\mathbf{0 5 . 0 9 . 2 0 1 7}$ \\
\hline District & $:$ & Dindigul & D/H: & $:$ & $\mathbf{2 2 . 1 2 . 2 0 1 7}$ \\
\hline
\end{tabular}

\begin{tabular}{|c|c|c|c|c|c|c|c|c|c|}
\hline \multirow[t]{2}{*}{ SI. No. } & \multirow[t]{2}{*}{ Treatments } & \multirow{2}{*}{$\begin{array}{c}\text { FYM } \\
\left(\mathrm{t} \mathrm{ha}^{-1}\right)\end{array}$} & \multicolumn{3}{|c|}{ Fertiliser doses $\left(\mathrm{kg} \mathrm{ha}^{-1}\right)$} & \multirow{2}{*}{$\begin{array}{c}\text { Grain } \\
\text { Yield } \\
\left(\mathrm{kg} \mathrm{ha}^{-1}\right)\end{array}$} & \multirow{2}{*}{$\begin{array}{c}\text { Per cent } \\
\text { achieve- } \\
\text { ment }\end{array}$} & \multirow{2}{*}{$\begin{array}{c}\text { RR } \\
\left(\mathrm{kg} \mathrm{kg}^{-1}\right)\end{array}$} & \multirow[t]{2}{*}{$\mathbf{B C R}$} \\
\hline & & & $\mathbf{F N}$ & $\mathrm{FP}_{2} \mathrm{O}_{5}$ & $\mathrm{FK}_{2} \mathrm{O}$ & & & & \\
\hline $\mathbf{T}_{1}$ & Blanket (100 \% RDF) & - & 250 & 75 & 75 & 7910 & - & 10.82 & 1.86 \\
\hline $\mathbf{T}_{2}$ & Blanket +FYM @ $12.5 \mathrm{t} \mathrm{ha}^{-1}$ & 12.5 & 298 & 99 & 107 & 9375 & - & 11.49 & 2.03 \\
\hline $\mathbf{T}_{3}$ & STCR-NPK alone-9.0 $\mathrm{t} \mathrm{ha}^{-1}$ & - & 218 & 108 & 79 & 9015 & 100.2 & 13.41 & 2.04 \\
\hline $\mathbf{T}_{4}$ & STCR-NPK alone-10.0 $\mathrm{t} \mathrm{ha}^{-1}$ & - & 256 & 123 & 97 & 9780 & 97.8 & 13.02 & 2.13 \\
\hline $\mathrm{T}_{5}$ & STCR-NPK alone-11.0 $\mathrm{t} \mathrm{ha}^{-1}$ & - & 293 & 137 & 115 & 10640 & 96.7 & 12.95 & 2.22 \\
\hline $\mathrm{T}_{6}$ & STCR-IPNS-9.0 $\mathrm{t} \mathrm{ha}^{-1}$ & 12.5 & 170 & 84 & 47 & 9280 & 103.1 & 14.07 & 2.08 \\
\hline $\mathbf{T}_{7}$ & STCR-IPNS-10.0 $\mathrm{t} \mathrm{ha}^{-1}$ & 12.5 & 208 & 99 & 65 & 10125 & 101.3 & 13.75 & 2.17 \\
\hline $\mathrm{T}_{8}$ & STCR-IPNS-11.0 $\mathrm{t} \mathrm{ha}^{-1}$ & 12.5 & 245 & 113 & 83 & 11060 & 100.5 & 13.72 & 2.28 \\
\hline $\mathrm{T}_{9}$ & Farmer's Practice & 0 & 225 & 95 & 0 & 6955 & - & 10.54 & 1.56 \\
\hline $\mathbf{T}_{10}$ & Absolute control & 0 & 0 & 0 & 0 & 3582 & - & - & 1.09 \\
\hline & & & & \multicolumn{2}{|c|}{ SEd } & 264 & & & \\
\hline & & & & \multicolumn{2}{|c|}{$\mathrm{CD}(\mathrm{P}=0.05)$} & 555 & & & \\
\hline
\end{tabular}

*maintenance dose

\begin{tabular}{l|l|l|l|}
\hline Fertilizer Prescription equations & \multicolumn{3}{|c|}{ Major Nutrients $\left(\mathrm{kg} \mathrm{ha}^{-1}\right)$} \\
\hline $\mathrm{FN}=3.78 \mathrm{~T}-0.78 \mathrm{SN}-0.890 \mathrm{~N}$ & $\mathrm{KMnO}_{4}-\mathrm{N}$ & $:$ & 185 \\
\hline $\mathrm{FP}_{2} \mathrm{O}_{5}=1.47 \mathrm{~T}-2.02 \mathrm{SP}-0.910 \mathrm{P}$ & Olsen-P & $:$ & 13 \\
\hline $\mathrm{FK}_{2} \mathrm{O}=1.79 \mathrm{~T}-0.14 \mathrm{SK}-0.620 \mathrm{~K}$ & $\mathrm{NH}_{4} \mathrm{OAc}-\mathrm{K}$ & $:$ & 550 \\
\hline
\end{tabular}


Table.3 Results of validation experiment on Maize

\begin{tabular}{|c|c|c|c|c|c|}
\hline \multicolumn{6}{|c|}{ Location } \\
\hline Village & : & Bathrakalipuram & Hybrid & : & TNAU Maize Hybrid CO 6 \\
\hline Block & : & Bodi & Soil & : & Pilamedu series (Typic Haplustert) \\
\hline Taluk & : & Bodi & $\mathrm{D} / \mathrm{P}:$ & : & 16.09 .2017 \\
\hline District & : & Theni & D/H: & : & 04.01 .2018 \\
\hline
\end{tabular}

\begin{tabular}{|c|c|c|c|c|c|c|c|c|c|}
\hline \multirow[t]{2}{*}{ Sl. No. } & \multirow[t]{2}{*}{ Treatments } & \multirow{2}{*}{$\begin{array}{c}\text { FYM } \\
\left(\mathrm{tha}^{-1}\right)\end{array}$} & \multicolumn{3}{|c|}{ Fertiliser doses (kg ha $\left.{ }^{-1}\right)$} & \multirow{2}{*}{$\begin{array}{c}\text { Grain } \\
\text { Yield } \\
\left(\mathrm{kg} \mathrm{ha}^{-1}\right)\end{array}$} & \multirow{2}{*}{$\begin{array}{c}\text { Per cent } \\
\text { achieve- } \\
\text { ment }\end{array}$} & \multirow{2}{*}{$\begin{array}{c}\text { RR } \\
\left(\mathrm{kg} \mathrm{kg}^{-1}\right)\end{array}$} & \multirow[t]{2}{*}{$\mathbf{B C R}$} \\
\hline & & & FN & $\mathrm{FP}_{2} \mathrm{O}_{5}$ & $\mathrm{FK}_{2} \mathrm{O}$ & & & & \\
\hline $\mathrm{T}_{1}$ & Blanket (100 \% RDF) & - & 250 & 75 & 75 & 7845 & - & 10.88 & 1.84 \\
\hline $\mathrm{T}_{2}$ & Blanket +FYM @ $12.5 \mathrm{t} \mathrm{ha}^{-1}$ & 12.5 & 298 & 98 & 106 & 9392 & - & 11.75 & 2.03 \\
\hline $\mathbf{T}_{3}$ & STCR-NPK alone-9.0 $\mathrm{t} \mathrm{ha}^{-1}$ & - & 203 & 108 & 79 & 8982 & 99.8 & 14.07 & 2.05 \\
\hline $\mathrm{T}_{4}$ & STCR-NPK alone-10.0 $\mathrm{tha}^{-1}$ & - & 241 & 123 & 97 & 9755 & 97.6 & 13.58 & 2.13 \\
\hline$T_{5}$ & STCR-NPK alone-11.0 $\mathrm{tha}^{-1}$ & - & 279 & 138 & 115 & 10560 & 96.0 & 13.28 & 2.21 \\
\hline $\mathrm{T}_{6}$ & STCR-IPNS-9.0 $\mathrm{t} \mathrm{ha}^{-1}$ & 12.5 & 155 & 85 & 48 & 9262 & 102.9 & 14.79 & 2.08 \\
\hline $\mathbf{T}_{7}$ & STCR-IPNS-10.0 $\mathrm{t} \mathrm{ha}^{-1}$ & 12.5 & 193 & 100 & 66 & 10070 & 100.7 & 14.26 & 2.17 \\
\hline $\mathrm{T}_{8}$ & STCR-IPNS-11.0 $\mathrm{t} \mathrm{ha}^{-1}$ & 12.5 & 231 & 115 & 84 & 10978 & 99.8 & 14.07 & 2.27 \\
\hline $\mathrm{T}_{9}$ & Farmer's Practice & 0 & 230 & 100 & 0 & 6880 & - & 10.26 & 1.53 \\
\hline$T_{10}$ & Absolute control & 0 & 0 & 0 & 0 & 3495 & - & - & 1.07 \\
\hline & & & & \multicolumn{2}{|c|}{ SEd } & 243 & & & \\
\hline & & & & \multicolumn{2}{|c|}{$\mathrm{CD}(\mathrm{P}=0.05)$} & 511 & & & \\
\hline
\end{tabular}

*maintenance dose

\begin{tabular}{|l|l|l|l|}
\hline Fertilizer Prescription equations & \multicolumn{3}{|c|}{ Major Nutrients $\left(\mathrm{kg} \mathrm{ha}^{-1}\right)$} \\
\hline $\mathrm{FN}=3.78 \mathrm{~T}-0.78 \mathrm{SN}-0.890 \mathrm{~N}$ & $\mathrm{KMnO}_{4}-\mathrm{N}$ & $:$ & 175 \\
\hline $\mathrm{FP}_{2} \mathrm{O}_{5}=1.47 \mathrm{~T}-2.02 \mathrm{SP}-0.910 \mathrm{P}$ & Olsen-P & $:$ & 12 \\
\hline $\mathrm{FK}_{2} \mathrm{O}=1.79 \mathrm{~T}-0.14 \mathrm{SK}-0.620 \mathrm{~K}$ & $\mathrm{NH}_{4} \mathrm{OAc}-\mathrm{K}$ & $:$ & 584 \\
\hline
\end{tabular}


Table.4 Range and mean values of validation experiments on maize

\begin{tabular}{|c|c|c|c|c|c|c|c|c|}
\hline \multirow[t]{2}{*}{ Sl. No } & \multirow[t]{2}{*}{ Treatments } & \multicolumn{3}{|c|}{ Fertiliser doses $\left(\mathrm{kg} \mathrm{ha}^{-1}\right)$} & \multirow{2}{*}{$\begin{array}{l}\text { Mean Grain } \\
\text { yield ( } \mathrm{kg} \\
\mathrm{ha}^{-1} \text { ) }\end{array}$} & \multirow{2}{*}{$\begin{array}{l}\text { Per cent } \\
\text { achievement }\end{array}$} & \multirow{2}{*}{$\underset{\left(\mathrm{kg} \mathrm{kg}^{-1}\right)}{\mathbf{R R}}$} & \multirow[t]{2}{*}{ BCR } \\
\hline & & $\mathbf{F N}$ & $\mathrm{FP}_{2} \mathrm{O}_{5}$ & $\mathrm{FK}_{2} \mathrm{O}$ & & & & \\
\hline $\mathbf{T}_{1}$ & Blanket (100 \% RDF) & 250 & 75 & 75 & 7927 & - & 10.96 & 1.84 \\
\hline $\mathbf{T}_{2}$ & $\begin{array}{l}\text { Blanket + FYM@ } 12.5 \\
\mathrm{t} \mathrm{ha}^{-1}\end{array}$ & 250 & 75 & 75 & 9419 & - & 11.67 & 2.03 \\
\hline $\mathbf{T}_{\mathbf{3}}$ & $\begin{array}{l}\text { STCR-NPK alone-9.0 t } \\
\mathrm{ha}^{-1}\end{array}$ & $186-218$ & 108 & $79-108$ & 9036 & 100.4 & 13.77 & 1.88 \\
\hline $\mathbf{T}_{4}$ & $\begin{array}{l}\text { STCR-NPK alone-10.0 t } \\
\mathrm{ha}^{-1}\end{array}$ & $224-256$ & 123 & $97-126$ & 9807 & 98.1 & 13.33 & 2.11 \\
\hline $\mathbf{T}_{\mathbf{5}}$ & $\begin{array}{l}\text { STCR-NPK alone-11.0 t } \\
\text { ha }^{-1}\end{array}$ & $261-293$ & $137-138$ & $115-144$ & 10650 & 96.8 & 13.16 & 2.20 \\
\hline $\mathbf{T}_{6}$ & STCR-IPNS-9.0 $\mathrm{t} \mathrm{ha}^{-1}$ & $140-170$ & $82-85$ & $47-75$ & 9304 & 103.4 & 14.44 & 2.07 \\
\hline $\mathbf{T}_{7}$ & STCR-IPNS-10.0 $\mathrm{t} \mathrm{ha}^{-1}$ & $178-208$ & $97-100$ & $65-93$ & 10145 & 101.5 & 14.05 & 2.16 \\
\hline $\mathbf{T}_{8}$ & STCR-IPNS-11.0 $\mathrm{t} \mathrm{ha}^{-1}$ & $215-245$ & $112-115$ & $83-111$ & 11075 & 100.7 & 13.95 & 2.26 \\
\hline$T_{9}$ & Farmer's Practice & $225-240$ & $75-100$ & 0 & 6938 & - & 10.56 & 1.69 \\
\hline $\mathbf{T}_{10}$ & Absolute control & 0 & 0 & 0 & 3541 & - & - & 1.07 \\
\hline
\end{tabular}

Major Nutrients $\left(\mathrm{kg} \mathrm{ha}^{-1}\right)$

\begin{tabular}{|l|l|l|}
\hline $\mathrm{KMnO}_{4}-\mathrm{N}$ & $:$ & $175-198$ \\
\hline Olsen-P & $:$ & $12-13$ \\
\hline $\mathrm{NH}_{4} \mathrm{OAc}-\mathrm{K}$ & $:$ & $380-584$ \\
\hline
\end{tabular}


Target yield equations generated from STCRIPNS technology ensured not only sustainable crop production but also economized use of costly fertilizer inputs (Mahajan et al., 2013). Farmer's practice recorded relatively lower yield and response ratio as compared to blanket and STCR treatments while the STCR-IPNS treatments recorded the highest per cent achievement, response ratio and BCR over farmer's practice (Table 4).

To conclude, the per cent achievement of the targeted yield of all the three validation experiments was within $+/-10 \%$ variation proving the validity of the fertiliser prescription equations for prescribing fertilizer doses for hybrid maize on Vertisol. The mean grain yield of hybrid maize from three validation experiments indicated that STCR-IPNS- $11 \mathrm{t} \mathrm{ha}^{-1}$ was found to record higher grain yield over all other treatments. All STCR-IPNS treatments recorded relatively higher response ratio and BCR over STCR treatments. The highest BCR was recorded in STCR-IPNS-11t ha ${ }^{1}$ andthereforethe fertilizer prescription equations developed for hybrid maize under IPNS can be recommended for black calcareous soils (Typic Haplustert) of Tamil Nadu for achieving yield target of $11 \mathrm{t} \mathrm{ha}^{-1}$ with sustained soil fertility.

\section{References}

Coumaravel, K., R. Santhi and S. Maragatham. 2013. Soil test crop response correlation studies under integrated plant nutrition system for hybrid maize on an Alfisol. Madras Agric. J., 100(7-9):660-664.

Debarati Bhaduri and Poonam Gautam., 2013. Optimization and validation of targeted yield equation based fertilizer doses under INM for wheat in TARAI region of Uttarakhand, India. Indian J. Agric. Res. 47(1): 16-25.
Gittinger, J. Price. 1982. Economic analysis of agricultural projects. Economic Development Institute of the World Bank. Johns Hopkins University Press. Baltimore, London.

Hanway, J.J. and Heidal H. (1952). Soil analysis methods as used in Iowa State College. Agriculture Bulletin.57:1-13.

Kumar, R., K. Srinivas and N. Sivaramane (2013), Assessment of the maize situation, outlook and investment opportunities in India. Country Report Regional Assessment Asia (MAIZECRP). 1-2.

Mahajan, G.R., R.N. Pandey., S.C. Datta. And S.C. Dinesh (2013).Soil test based fertilizer recommendation of nitrogen, phosphorous and sulphur in wheat (Triticum aestivum L.) in an alluvial soils. Intl.J.Agric.Env.Biotech, 6:271281.

Manish Singh, Y.V. Singh, S.K. Singh, Pradip Dey, L.K. Jat and Ram, R.L. 2017. Validation of Soil Test and Yield Target based Fertilizer Prescription Model for Rice on Inceptisol of Eastern Zone of Uttar Pradesh. Int.J.Curr.Microbiol. App.Sci. 6(2): 406-415.

Olsen, S.R, Cole, C.V and Watanabe. F.S. (1954). Estimation of Available Phosphorous in Soils by Extraction with Sodium Bicarbonate. Pp. 939. Circular US Dept. of Agriculture, 1400 Independence Ave. S.W., Washington DC.

Ramamoorthy. B, RL Narasimham, RS Dinesh. $1967 . \quad$ Fertilizer recommendations based on fertilizer applications for specific yield targets of Sonara-64., Indian farming: 17(5): 4345.

Santhi. R., A. Bhaskaran and R. Natesan. 2011. Integrated fertilizer prescriptions for beetroot through Inductive cum targeted yield model on an Alfisol. 
Commun. Soil. Sci. Pl. Analysis, 42: 1905-1912.

Sellamuthu, K.M, R. Santhi, S. Maragatham and P. Dey (2015). Validation of soil test and yield target based fertilizer prescription model for wheat on Vertisol. Research on crops, 16 (1):5358.

Sharma, V.K., R.N. Pandey and B.M.Sharma.2015.studies on long term impact of STCR based. Integrated fertilizer use on pearl millet (Pennisetum glaucum)-wheat (Triticum aestivum) cropping system in semi-arid condition of India. J. Environ. Bio, 36(1): 241-247.

Singh. M., B.S. Dwivedi and S.P. Datta. 2012. Integrated nutrient management for enhancing productivity, nutrient use efficiency and environmental quality. In: Soil Science in the service of nation, Pp. 55-57. ISSS, New Delhi.

Subbiah, B.V. and G.L. Asija. (1956). A rapid procedure for estimation of available nitrogen in soils. Curr. Sci., 25: 259260.

\section{How to cite this article:}

Suresh, R. and Santhi, R. 2018. Validation of Soil Test and Yield Target Based Fertiliser Prescription Model for Hybrid Maize on Vertisol. Int.J.Curr.Microbiol.App.Sci. 7(09): 21312139. doi: https://doi.org/10.20546/ijcmas.2018.709.261 